\title{
Les élections présidentielles de 2007 à Timor Leste
} Recherche de changement et de sortie de crise dans un rééquilibrage politique

\section{Christine Cabasset-Semedo et Frédéric Durand}

\section{OpenEdition}

Journals

Édition électronique

URL : http://journals.openedition.org/echogeo/1164

DOI : $10.4000 /$ echogeo.1164

ISSN : 1963-1197

Éditeur

Pôle de recherche pour l'organisation et la diffusion de l'information géographique (CNRS UMR 8586)

Référence électronique

Christine Cabasset-Semedo et Frédéric Durand, « Les élections présidentielles de 2007 à Timor Leste», EchoGéo [En ligne], Sur le Vif, mis en ligne le 16 juillet 2007, consulté le 19 avril 2019. URL: http://journals.openedition.org/echogeo/1164; DOI : 10.4000/echogeo.1164

Ce document a été généré automatiquement le 19 avril 2019

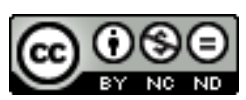

EchoGéo est mis à disposition selon les termes de la licence Creative Commons Attribution - Pas d'Utilisation Commerciale - Pas de Modification 4.0 International 


\title{
Les élections présidentielles de 2007 à Timor Leste
}

\author{
Recherche de changement et de sortie de crise dans un rééquilibrage \\ politique
}

\section{Christine Cabasset-Semedo et Frédéric Durand}

Après presque un quart de siècle d'occupation indonésienne (1975-1999) et trois ans d'administration par l'ONU, le Timor Leste est devenu indépendant le 20 mai 2002. Classé sur la liste des pays les moins avancés, engagé dans une phase de reconstruction et de construction nationale, le pays a déjà connu plusieurs crises. La plus récente, au premier semestre 2006, a été la plus violente et la plus étendue. Partie d'un conflit dans l'armée nationale, elle s'est propagée aux populations civiles et s'est muée en crise politique. La menace d'un embrasement en une guerre civile a provoqué l'arrivée, en mai 2006, d'une force multinationale de 3000 hommes. Les élections présidentielles de 2007, vues comme à «haut risque " dans ce contexte tendu, étaient particulièrement suivies par la communauté internationale. Néanmoins, au plan intérieur, la politique et les élections présidentielles est-timoraises sont marquées, en ce qui concerne les acteurs politiques, du signe du changement dans la continuité, du moins depuis la fin de l'époque coloniale portugaise.

2 D’un point de vue historique, le pays a connu quatre présidents depuis la décolonisation engagée par le Portugal au lendemain de la révolution des đillets (avril 1974). Le premier a été Francisco Xavier do Amaral, fondateur en mai 1974 de l'ASDT (Association Sociale Démocrate Timoraise), devenue quatre mois plus tard le Fretilin (Front Révolutionnaire pour l'Indépendance de Timor-Est). Le Fretilin avait pris le pouvoir de fait, en août 1975, après un coup d'État manqué de son rival de droite l'UDT (Union Démocratique Timoraise) ${ }^{1}$. Devant les multiples attaques de l'armée indonésienne en prélude à l'invasion officielle, Francisco Xavier do Amaral avait déclaré unilatéralement l'indépendance le 28 novembre 1975, devenant le premier président de la république démocratique de Timor Leste (Defert, 1992). À l'époque, José Ramos Horta, membre fondateur du Fretilin, avait été nommé ministre des Affaires étrangères. Il avait quitté le territoire quelques jours avant 
l'invasion du 7 décembre, pour chercher du soutien à l'étranger, ce qui lui a valu 24 ans d'exil forcé. Suite à des dissensions internes au Fretilin entré en résistance dans les montagnes, do Amaral a été destitué en septembre 1977 (Dunn, 1983), et remplacé par Nicolau Lobato son premier ministre, qui est devenu de facto le deuxième président, avant de mourir lors d'une offensive indonésienne en décembre 1978. La résistance timoraise, qui avait déjà connu de lourdes pertes, s'est reconstruite lorsque Xanana Gusmao en a pris la direction à partir de 1981, pour aboutir quelques années plus tard à la constitution d'une union nationale (civile et militaire) intégrant tous les secteurs de la société et mouvances politiques pro-indépendantistes. Cette union a été le noyau du CNRM (Conseil National de la Résistance Maubere) formé en 1988 pour réconcilier les anciens rivaux des années 1970 et devenu en 1998 le CNRT (Conseil National de la Résistance Timoraise).

Le référendum d'autodétermination d'août 1999 sous l'égide de l'ONU, avec 78,5\% des voix en faveur de l'indépendance, a mis fin à 24 ans d'occupation indonésienne ${ }^{2}$ et a scellé, avec la déclaration de l'indépendance du pays en mai 2002 (Durand, 2002), le retour sinon l'émergence, d'une démocratie à l'occidentale ${ }^{3}$. Alors que le pays était encore sous tutelle onusienne, deux élections ont eu lieu: pour la formation d'une Assemblée constituante en août 2001, qui ont donné $57 \%$ de suffrages au Fretilin, et pour les présidentielles en avril 2002 entre Xanana Gusmão et l'ancien premier président do Amaral, qui avait refondé l'ASDT (Durand, 2001). Xanana Gusmão, également membre du Fretilin dans les années 1970, avait quitté ce parti et pris la tête de la plate-forme commune du CNRT. En 2001, Xanana avait cependant préféré dissoudre le CNRT afin de ne pas interférer dans les jeux politiques. Personnage plus charismatique et surtout plus consensuel que do Amaral, Xanana avait été élu président le 14 avril 2002 avec $83 \%$ des voix, l'emportant largement dans tous les districts, sauf dans celui d'Aileu d'où était natif do Amaral.

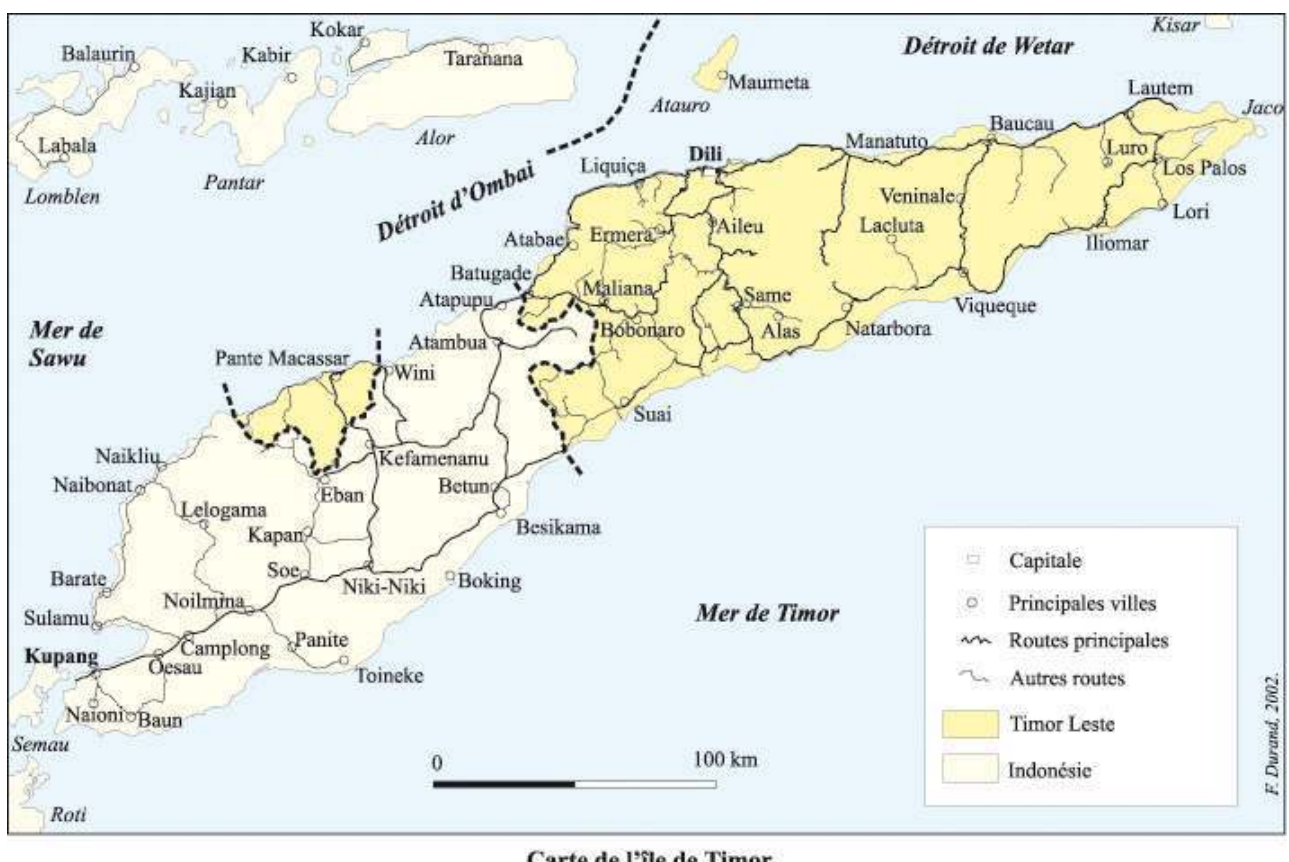

De 2002 à 2007, Xanana Gusmão a fait l'exercice d'un pouvoir présidentiel limité, plus symbolique que gestionnaire, dû au régime parlementaire (Constitution, 2001). C'est Mari Alkatiri, membre du Fretilin (et ministre des Affaires politiques dans le premier 
gouvernement de 1975) qui a assuré la fonction de Premier ministre. Au cours de ces cinq années, le soutien populaire au Fretilin a eu tendance à s'éroder en raison de crises liées au manque de changement, à des confrontations avec l'Église, importante dans ce pays qui compte $95 \%$ de catholiques (Durand, 2004) et à une rébellion de militaires de la partie occidentale du territoire. La crise de 2006 trouve en effet son origine dans le licenciement de presque 600 membres de l'armée nationale (F-FDTL), issus de l'ouest du pays, qui avaient quitté leur caserne parce qu'ils s'estimaient discriminés par rapport à ceux de l'extrême-est qui fournissent à l'armée le plus fort contingent et notamment les cadres. De militaire, ce conflit s'est étendu à la société civile, provoquant des tensions entre populations de l'ouest et de l'est. De fait, il existe un clivage ancien, mais qui n'est pas ethnique ni linguistique, entre les populations de l'ouest de Timor-Est (Kaladi/Loromonu) et ceux du grand-est (Firaku/Lorosae). Ce clivage s'est forgé à l'époque coloniale portugaise entre ceux de l'ouest proche de Dili et considérés comme plus «assimilés » et ceux de l'est, regardés comme plus «frustres ». Cette différence a été renforcée de 1975 à 1999, dans la mesure où l'essentiel de la résistance armée liée au Fretilin s'est concentrée à l'est, zone que l'armée indonésienne contrôlait moins. En 2006, les troubles qui ont résulté de ces tensions est/ouest ont provoqué la mort de 37 personnes, le déplacement de 150000 autres et ont rendu nécessaire la présence d'une force multinationale. Face à la mauvaise gestion de ces crises, le Premier ministre Fretilin, Mari Alkatiri, avait dû donner sa démission et céder la place à José Ramos Horta. Début 2007, un groupe de militaires et de policiers contestataires se cachait toujours dans les montagnes sous le commandement du major Alfredo Reinado.

5 Les élections présidentielles de 2007 se sont ainsi déroulées dans un contexte tendu, avec une volonté de rupture de la part de la population vis-à-vis du parti historique du Fretilin.

6 Francisco Guterres («Lu Olo»), originaire de Ossu (est du pays), est le président du Fretilin (55 députés sur 88). Sa force politique résidait dans son parcours de résistant puisqu'il est l'un des rares Timorais à être resté dans la guérilla pendant les 24 ans d'occupation, autant que dans le nom même du Fretilin toujours associé à la lutte pour l'indépendance. En outre, le parti ne pouvait plus guère être considéré comme marxiste, même si son empreinte historique l'y associait. En revanche, ses membres sont généralement considérés comme relevant plus de la mouvance lusophone et de réseaux tournés vers le Portugal, le Mozambique et l'Angola, ce qui attire peu la jeune génération.

7 José Ramos Horta, son principal rival, personnalité la plus connue sur la scène internationale (il fût notamment l'un des candidats pressentis au poste de secrétaire général de l'ONU en 2006) était de son côté auréolé de son image de défenseur du peuple timorais depuis 1975, ce qui lui avait valu de partager en 1996 le Prix Nobel de la Paix avec l'évêque de Dili, Monseigneur Carlos Ximenes Belo. Il bénéficiait en outre du soutien affiché du président sortant, qui ne souhaitait pas se représenter. Ses grandes qualités de polyglotte le rattachaient bien sûr au Portugal, mais aussi au monde anglo-saxon et notamment à l'Australie où il avait vécu une bonne partie de son exil.

8 Trois autres candidats étaient considérés comme des challengers sérieux, dans la mesure où leur parti était arrivé juste après le Fretilin (quoique avec seulement 7 à $8 \%$ chacun) lors des élections constitutionnelles de 2001. Francisco Xavier do Amaral se présentait une nouvelle fois au nom de l'ASDT avec le prestige de l'âge, même si ses 70 ans pouvaient jouer aussi en sa défaveur. Fernando de Araujo en revanche pouvait créer la surprise. Également connu sous le nom de "Lasama », de l'époque où il avait créé en 1988 le réseau clandestin Renetil (Résistance Nationale des Étudiants de Timor Leste), il avait été capturé par 
l'armée indonésienne en 1991 et emprisonné avec Xanana Gusmão de 1992 à 1998. Il incarne la «jeune génération" contemporaine de l'occupation indonésienne, en opposition à la « génération de 1975 » de l'élite politique au pouvoir. Son parti, le Parti Démocrate (PD) avait fait le $2^{\mathrm{e}}$ score en 2001. Il avait en outre obtenu le soutien de l'Église catholique, ainsi que celui d'Alfredo Reinado, le leader des militaires rebelles. Lucia Lobato, la seule femme candidate, se présentait au titre du Parti Social Démocrate (PSD), lancé en 2001 par Mário Carrascalão. Ce dernier, fondateur de l'UDT, le parti de droite des années 1970, était ensuite devenu gouverneur pendant l'occupation indonésienne. Bien que disposant d'une faible marge de manœuvre et obligé d'œuvrer en faveur de l'« intégration », il avait néanmoins osé défendre la population contre les abus de l'armée d'occupation. Les trois derniers candidats relevaient de composantes moins importantes: Manuel Tilman du Kota, un parti traditionaliste pour le retour des pouvoirs traditionnels; Avelino Coelho da Silva du PST (Parti Socialiste de Timor) et João Carrascalão pour l'UDT, le parti de droite, rival du Fretilin en 1974/1975.

Malgré des craintes et quelques incidents mineurs, le premier tour qui s'est tenu le 9 avril 2007 s'est déroulé dans le calme et dans le respect de la loi (SOMET, 2007). Comme toujours depuis 1999, les Timorais ont voté en masse avec 81,8 \% de votants. Les résultats ont été relativement serrés. Le Fretilin est arrivé en tête, mais avec $28 \%$ des voix, contre $22 \%$ pour Ramos Horta et 19 \% pour Fernando de Araujo (cf. tableau ci-dessous)

\begin{tabular}{|c|c|c|c|c|c|c|c|c|}
\hline & $\begin{array}{l}\text { Francisco } \\
\text { Guterres }\end{array}$ & $\begin{array}{c}\text { Jose Ramss } \\
\text { Horta }\end{array}$ & $\begin{array}{l}\text { Fernando } \\
\text { de Aratigo }\end{array}$ & $\begin{array}{l}\mathrm{FX} \text {. do } \\
\text { Amaral }\end{array}$ & $\begin{array}{l}\text { Lixia } \\
\text { Lobata }\end{array}$ & $\begin{array}{l}\text { Manuel } \\
\text { Tilman }\end{array}$ & $\begin{array}{l}\text { Avelino } \\
\text { Cootho }\end{array}$ & $\begin{array}{l}\text { Joâo } \\
\text { Camassalito }\end{array}$ \\
\hline Parti & Fretilin & . & PD & ASIDI: & PSD & Kota & PST & UDT \\
\hline $\begin{array}{l}\text { Score 2001 } \\
\text { Paricmentairss }\end{array}$ & $57,4 \%$ & - & $8.7 \%$ & $7.8 \%$ & $8,2 \%$ & $2.1 \%$ & $1.8 \%$ & $2,4 \%$ \\
\hline $\begin{array}{l}1=\text { tour } 2007 \\
\text { Prividantivilas }\end{array}$ & $27,9 \%$ & $21.8 \%$ & $19,2 \%$ & $14,4 \%$ & $8.9 \%$ & $4,1 \%$ & $2,1 \%$ & $1.7 \%$ \\
\hline
\end{tabular}

Sources: Durand (E). 2002 ; CNE-Timor 2007

11 Si l'on compare avec les données des élections parlementaires de 2001, le Fretilin est le plus fragilisé avec une perte de près de $30 \%$. Cela s'explique en partie par la concurrence de José Ramos Horta, qui se présentait sans véritable parti, mais avec le soutien du président sortant Xanana Gusmão. Ce dernier a refondé en 2007 le CNRT, avec un nouveau sens à ce sigle (Congrès National pour la Reconstruction de Timor). L'autre grand gagnant était le PD de Fernando de Araujo qui aurait pu accéder au $2^{\mathrm{e}}$ tour. Tous les autres partis ont amélioré leur score, sauf l'UDT sans doute encore entachée de l'image négative de 1975.

D'un point de vue régional, on peut noter un certain nombre de clivages. Le Fretilin est arrivé largement en tête dans les trois districts de l'est, d'où sont originaires les principaux chefs de la résistance, les cadres de l'armée actuelle ou des personnalités politiques comme « Lu Olo». 


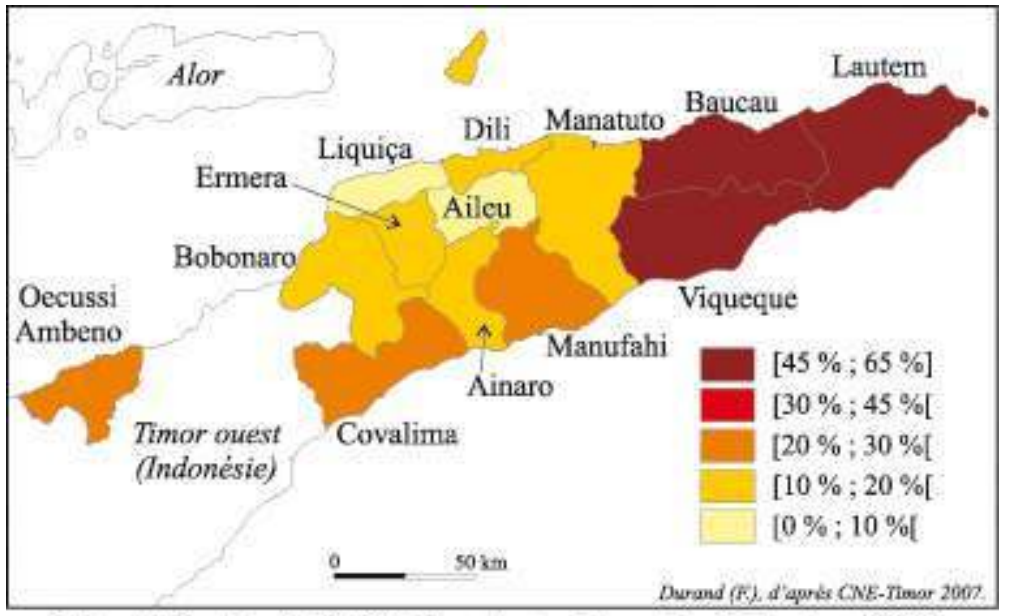

Carte 1: Résultats du FRETILIN au ler tour des présidentielles d'avril 2007

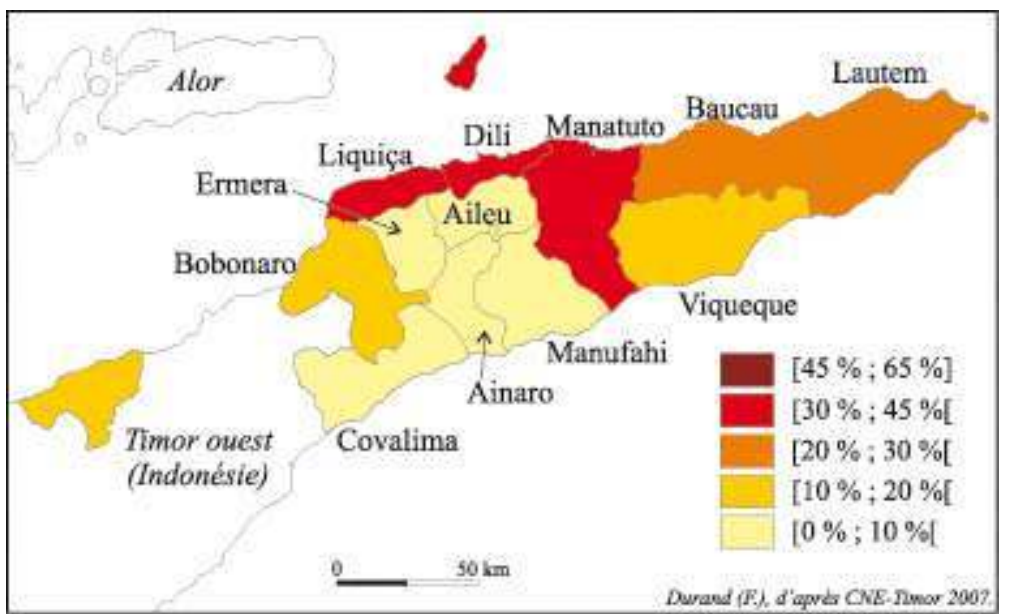

Carte 2 : Résultats de José Ramos Horta au ler tour des présidentielles d'avril 2007

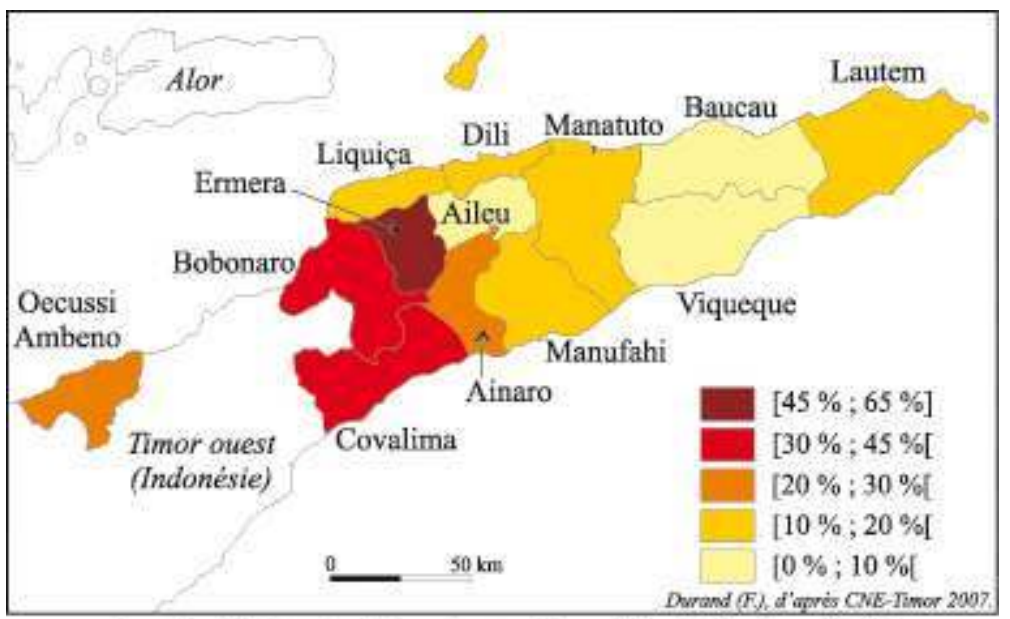

Carte 3 : Résultats du PD au ler tour des présidentielles d'avril 2007 


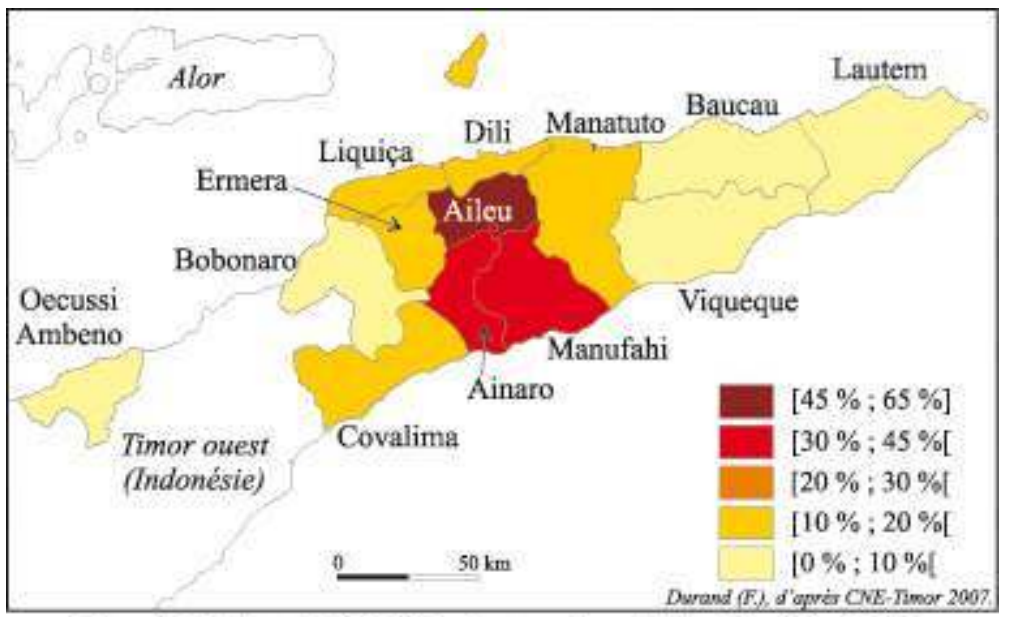

Carte 4: Résultats de l'ASDT au ler tour des présidentielles d'avril 2007

José Ramos Horta a surtout obtenu de bons résultats sur la côte occidentale, autour de Dili (carte 2) ; tandis que Fernando de Araujo l'emportait dans l'arrière pays de l'ouest et dans l'enclave d'Oecussi (carte 3). Francisco Xavier do Amaral a quant à lui surtout dominé à Aileu son district d'origine et dans une moindre mesure au centre de la côte sud (carte 4). On notera également des scores non négligeables de Lucia Lobato dans trois districts de l'ouest (Oecussi : $22 \%$; Bobonaro : 19\%; Liquiça : $17 \%$ ) et de Manuel Tilman à Ainaro (25\%).

Ce scrutin du premier tour a donc été marqué par un fort régionalisme et des différences appuyées dans les votes en fonction des ancrages locaux des candidats. Le même phénomène se retrouve au $2^{\mathrm{e}}$ tour de la présidentielle qui a eu lieu le 9 mai 2007. La participation a été légèrement moindre qu'au premier tour, mais encore élevée, avec $78,8 \%$ des inscrits. José Ramos Horta, qui avait obtenu le soutien de Fernando de Araujo $\mathrm{PD}$ ) et de tous les autres partis (à l'exception du Kota) très implantés dans la région ouest la plus densément peuplée, a largement remporté ces élections avec $69,2 \%$ des suffrages. Francisco Guterres pour le Fretilin est, quant à lui, arrivé en tête dans les trois districts de l'extrême-est avec respectivement 68,67 et $51 \%$ à Viqueque, Baucau et Lautem, confirmant la tendance du premier tour (cartes 5 et 6 ).

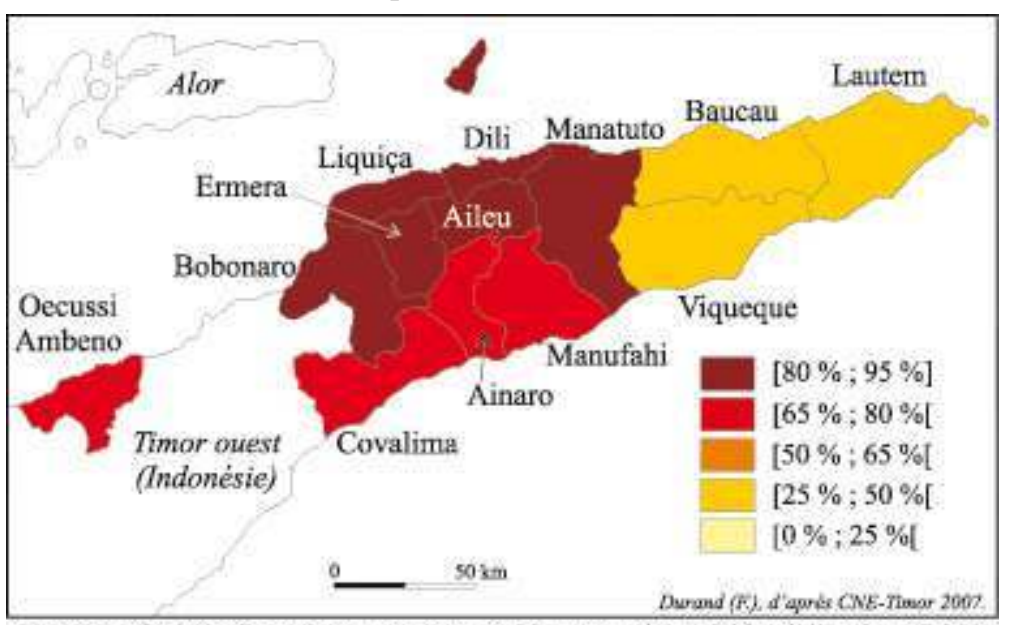

Carte 5 : Résultats de José Ramos Horta au 2ème tour des présidentielles de mai 2007 


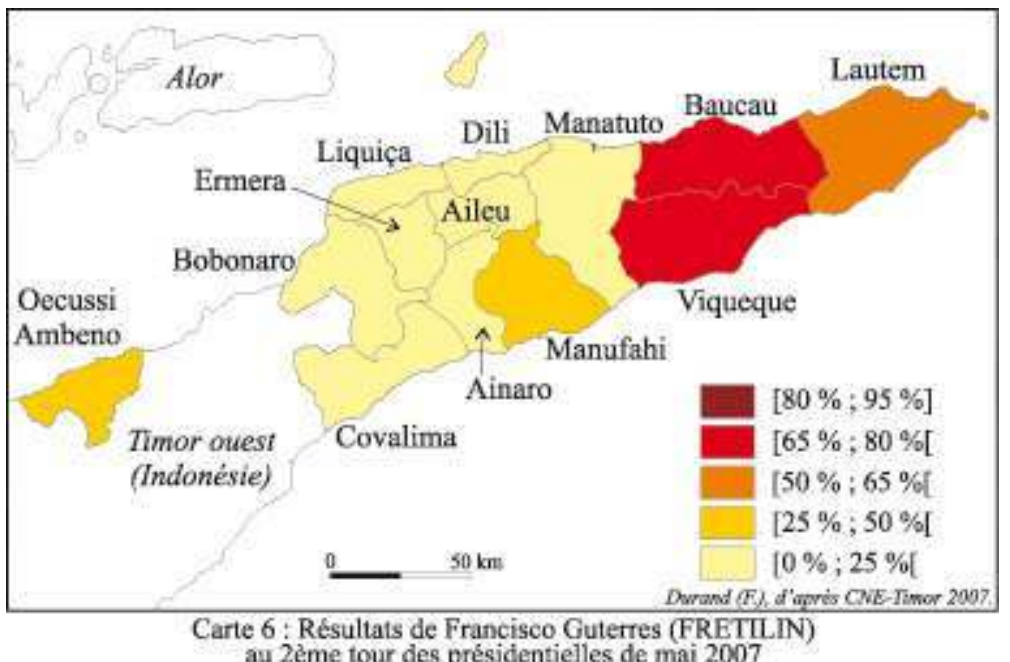

Les élections législatives qui ont suivi, le 30 juin 2007, ont confirmé cette tendance. Le Fretilin est arrivé en tête, mais avec seulement $29 \%$, devant le CNRT : $24 \%$, l'ASDT-PSD : $16 \%$ et le PD : $11 \%$. Sans majorité absolue, les différents partis sont obligés de s'associer en coalitions pour pouvoir revendiquer la formation d'un gouvernement.

Au final, ces élections indiquent une volonté de changement vis-à-vis du Fretilin qui s'est essoufflé après cinq années de pouvoir. Les Timorais ont néanmoins préféré une certaine continuité, en jouant le rééquilibrage politique et en élisant José Ramos Horta à la présidence, un pionnier de l'indépendance, qui plus est de stature internationale. La mission majeure du nouveau président sera de chercher un consensus permettant de dépasser les clivages régionaux et de répondre aux attentes d'amélioration du niveau de vie des habitants.

\section{BIBLIOGRAPHIE}

CAVR, 2006, Chega! The report of the Commission for the Reception, truth and Reconciliation in Timor Leste, $2500 \mathrm{p}$.

http://www.cavr-timorleste.org/chegaReport.htm

Constitution of Democratic Republic of Timor Leste, 2001, 68 p.

http://www.timor-leste.gov.tl/constitution/constitution.htm

Defert G., 1992, Timor-Est, le génocide oublié, L’Harmattan, Paris, 323 p.

Dunn J., 1983, Timor, A People Betrayed, Jacandra Press, Milton Queensland, 402 p.

Durand F., 2006, Timor 1250-2005, 750 ans de cartographie et de voyages, Arkuiris-IRASEC, Toulouse et Bangkok, $520 \mathrm{p}$.

Durand F., 2004, Catholicisme et Protestantisme dans l'île de Timor, 1556-2003, Arkuiris-IRASEC, Toulouse, $240 \mathrm{p}$. 
Durand F., 2002, Timor Est, pays au carrefour de l'Asie et du Pacifique, PUMLV-IRASEC, Toulouse, $208 \mathrm{p}$.

Durand F., 2001, « Timor Lorosa'e 1930-2001 : partis politiques et processus électoraux à hauts risques » in Aséanie 8, p 103-126.

SOMET, 2007, Report on the first round of the Timor-Leste Presidential Elections, ETAN, 15 p.

\section{NOTES}

1. Fondée notamment par les frères Carrascalão.

2. Qui s'est soldée par 100000 à 180000 morts, les pics de mortalité se situant de 1975 à 1984 (morts violentes, famines...) et, proportionnellement en 1999, après l'annonce des résultats du referendum. Cf. Rapport du CAVR, 2006.

3. On signalera à ce propos que les chefferies est-timoraises connaissaient des formes traditionnelles de démocraties, au point que certains observateurs du XIX ${ }^{\mathrm{e}}$ siècle ont parlé de « républiques aristocratiques » (Cf. Durand (F.), 2006.

\section{RÉSUMÉS}

En avril-mai 2007 des élections présidentielles se sont tenues à Timor-Est. Ce pays, indépendant depuis mai 2002, a connu une série de crises au cours des dernières années, qui a abouti à une remise en question du rôle dominant du Fretilin, le parti historique de la lutte contre l'invasion indonésienne et pour l'indépendance, au pouvoir depuis 2002. Le premier tour a été serré avec trois candidats entre 19 et $28 \%$. Au deuxième tour, qui opposait Lu-Olo, candidat du Fretilin à José Ramos Horta, ancien Premier ministre, co-lauréat du Prix Nobel de la Paix, c'est ce dernier qui a finalement été élu avec $69 \%$ des suffrages. Les élections législatives qui se sont tenues le mois suivant, en juin, ont confirmé l'affaiblissement du Fretilin et le rééquilibrage politique.

In April-May 2007 presidential elections were held in East-Timor. Since independence (2002), the country crossed several crisis, calling into question the leading role of the Fretilin, the historical party which fought against Indonesia invasion and for independance. The first round of the ballot was tight with three candidates between 19 and 28\%. At the end of the second round, opposing Lu-Olo, Fretilin's candidate to José Ramos Horta, former Prime minister, co-Nobel Peace Laureate in 1996, this last has been elected with $69 \%$ of the votes. The legislative elections which were held the following month, in June, confirmed the Fretilin weakening and the political rebalancing.

Em abril-maio de 2007 havia eleições presidenciais em Timor Leste. Independente desde maio de 2002, o país conheceu uma série de crises no decorrer destes últimos anos, que acabaram por abalar o papel dominante da Fretilin, partido histórico da luta contra a invasão indonésia e pela independência. A primeira volta foi apertada, com três candidatos obtendo entre 19 e $28 \%$ dos votos. Na segunda volta, que opunha Lu-Olo, candidato do Fretilin, a José Ramos Horta, exPrimeiro ministro, co-laureado do Prémio Nobel da Paz, este ùltimo acabou por ser eleito com 
$69 \%$ dos votos. As eleições legislativas que se realizaram no mês seguinte, em Junho, confirmaram o enfraquecimento da Fretilin e o reequilíbrio político.

\section{AUTEURS}

\section{CHRISTINE CABASSET-SEMEDO}

Christine Cabasset-Semedo, christine.cabasset@free.fr, est docteur en géographie de l'Université Paris Sorbonne (Paris-IV). Ses deux dernières publications sont Timor Leste : quelles stratégies de développement touristique dans une situation de postconflit? In Actes du séminaire Crises et violences dans l'aire Asie-Pacifique, Paris, Presses de l'Université Paris IV (PUPS), 2007 (A paraître) ; Timor Leste : l'écotourisme comme première modalité d'interface. In Actes des Journées de Géographie Tropicale (par l'Université des Antilles et Guyane), 2007 (A paraître).

\section{FRÉDÉRIC DURAND}

Frédéric Durand, durand@univ-tlse2.fr, est maître de conférences-HDR et membre du laboratoire CNRS CASE (Centre Asie du Sud-Est) UMR 8170 équipe LASEMA., Ses deux dernières sont publications Timor 1250-2005, 750 ans de cartographie et de voyages, Arkuiris-IRASEC, 2006 ; Catholicisme et Protestantisme dans l'île de Timor, 1556-2003, construction d'une identité chrétienne et engagement politique contemporain, Arkuiris-IRASEC, 2004. 